\title{
Sharing intermediate datasets from systematic reviews
}

\author{
Tiago Lubiana $\cdot \underline{0000-0003-2473-2313} \cdot$ School of Pharmaceutical Sciences, University of São Paulo; Ronin \\ Institute
}

Olavo B. Amaral $\cdot \underline{0000-0002-4299-8978} \cdot$ Institute of Medical Biochemistry Leopoldo De Meis, Federal University of Rio de Janeiro

Kleber Neves $\cdot \underline{0000-0001-9519-4909} \cdot$ Institute of Medical Biochemistry Leopoldo De Meis, Federal University of Rio de Janeiro

\section{Abstract}

Thousands of systematic reviews are published every year. To conduct a systematic review, researchers spend hours of work extracting data from and about the individual papers that are included. This step often leads to the production of spreadsheets with structured data and metadata about scientific publications, which are the basis for later steps in the project. These datasets will often remain hidden in local folders and unavailable, despite the time invested in producing them and their potential value. We argue that this is a great opportunity, where a small amount of additional planning to make these intermediate datasets publicly available can be valuable to the scientific community, while also allowing authors to be cited and recognized.

When one performs a systematic search and records information from the literature, curated tables are usually generated. These tables organize information scattered across the literature and come in many flavours: a list of gene expression datasets related to a disease, a set of articles united by the same methodology, a collection of protocols used to grow a cell line of interest, and so on. In systematic reviews, articles considered for inclusion are typically screened, and various types of metadata are logged into intermediary curation tables, such as information on the publication (e.g. journal, authors), methods (e.g. type of study, outcomes) and/or results (e.g. effect estimates). However, the data collection achieved after many hours of work is frequently relegated to forgotten folders and does not always become part of the final publication.

Take an example from our own work: two of the authors work in a large project that aims to replicate biomedical experiments published in articles originating from Brazil [1]. To select experiments for replication, a full-text search of 20 years of biomedical literature from the country was conducted, with a focus on finding articles using specific experimental methods. As a result, we obtained information about methods (e.g. RT-PCR or cell viability assay) and experimental models (e.g. cell line or species) used in the 195 articles retrieved in the search -, information that was extracted and checked by two separate researchers. We used these data to select the sample of experiments to be replicated, and the information regarding these selected experiments will be made public when the project is finished. However, despite its added value, most of the data obtained in this step, which also comprises the non-selected articles, remained hidden in a folder in the authors' hard drive.

These data represent dozens of hours of specialized work - one survey estimates that it takes at least a third of the total time in a typical systematic review [2] -, with a concrete result - the intermediate dataset containing the metadata about the articles. Nevertheless, they remain unusable if they are not shared. There are tools, however, to make these small intermediate datasets usable with little effort and unlock their potential. We thus advocate for sharing of the small (or not so small) curated datasets made by every researcher in their literature reviews. We argue that this can provide a 
new knowledge resource for life scientists while also benefiting researchers' careers.

Large curation projects, such as UniProt and Gene Ontology, hire "biocurators" to organize the knowledge and have become core tools in the modern life sciences. Personal curations performed by independent scientists, while less visible, are also high-quality data embodying the perspectives of experts. Similarly to the collective value of small-scale experimental datasets [3] , this long tail of small-scale tables of structured information represents a wealth of shareable knowledge.
First of all, this work has value: gathering data from multiple sources into a usable dataset is a skill in itself, and other researchers can have a use for your curated data, even if it is not perfect. Compiling information from the literature for a research project is no small feat, and can save many hours of work by other people. And even if you don't see an immediate use for the data, other researchers might have unexpected applications for it.

Second, your work will be findable by anyone who wants to work with the data. Google Datasets [4], for instance, makes it very easy to find this kind of data (see Box).

\section{How to share your curated datasets}

Our proposal tries to balance the cost of making intermediate datasets available with the value for both the researchers who perform data curation and the scientific community at large [4]. We thus focus on systematic reviews, where data curation is already a necessary step and data provenance sources used and curation methods - will likely be documented in the published article. Because the dataset is a byproduct of the research project, the only added step - if data is collected in a structured fashion - is making the dataset available, as researchers will probably already have it as a table (say, in CSV or Excel format) or database (such as a Microsoft Access or Libre Office Base file). In any case, the same steps can be followed for other types of structured datasets. While we give general directions here, we also provide more detailed step-by-step tutorials elsewhere (https://no-budget-science.github.io/en/table to zenodo).

1. Make it findable: nowadays, there are many possibilities for making datasets available with a DOI. While we recommend Zenodo []ㅡ, any DOI-providing repository, like figshare [ 6 ] or Dryad [7] will work. As we mentioned above, curation of datasets is specialized work, and being citable makes it easy for this work to be recognized. Another benefit is that Zenodo datasets are automatically indexed by data-specific search engines, such as Google Datasets, which makes them findable by interested researchers.

2. Make it reusable: Describe datasets with enough information to facilitate reuse. A simple solution is to provide a data dictionary - a thorough description of what is contained in each column of the dataset. Whenever possible, use unique identifiers for data (e.g., identify scientific articles by their DOI, instead of a full citation). [8]

3. Make it interoperable: if you feel comfortable, an extra step is to add the data to open knowledge repositories, such as Wikidata [9], while referencing your publicly available table. This makes your curation available in a standard format and immediately integrates it with data from many other sources. In any case, make sure the data is available under an open license, preferably CCO, as it empowers others to reuse it. [1이] 
This makes science a more communal and shared endeavour and helps bring in scientists who otherwise could not participate in it [11]. Moreover, you (or someone in your research group) are the most likely person to need the dataset again in the future. And in 5 years time, when you need it, it may come in handy to find your curated dataset on Google instead of spending hours sifting through old emails, Dropbox folders or hard drives.

You can also be rewarded in other ways. A published table can receive a $\mathrm{DOI}$ and become citable, which means you get recognition for your work. Empirical research suggests that publications whose datasets are open are cited more often [12], and openness is increasingly recognized as an essential aspect of scientific work [13]. Publishers and funders recognize the importance of open data and are moving in that direction with their policies [14].

Publicizing intermediate datasets brings a lot of value for little extra work - but only if this is planned in advance. Coming back to our example, the referred intermediate table is now available [15]. However, releasing it publicly came as an afterthought. As a consequence, it took hours of work to gather the data from various files and spreadsheets and then double-check and standardize the information. Much of this work could have been avoided had we followed a standard format and had a data management and preservation plan established from the start.

Nevertheless, we can already grasp the benefits of sharing our intermediate dataset. All data published under open licenses like Creative Commons BY and CCO [16] become available for integration with larger projects. Thus, it became possible to add the information from our published dataset to Wikidata, the Wikipedia sister project for structured knowledge [17]. Once in Wikidata, the information became integrated within a larger body of data, allowing it to be harnessed by programmers. As an example, Figure 1 maps the affiliations of authors of articles screened by the Brazilian Reproducibility Initiative that used RT-PCR.

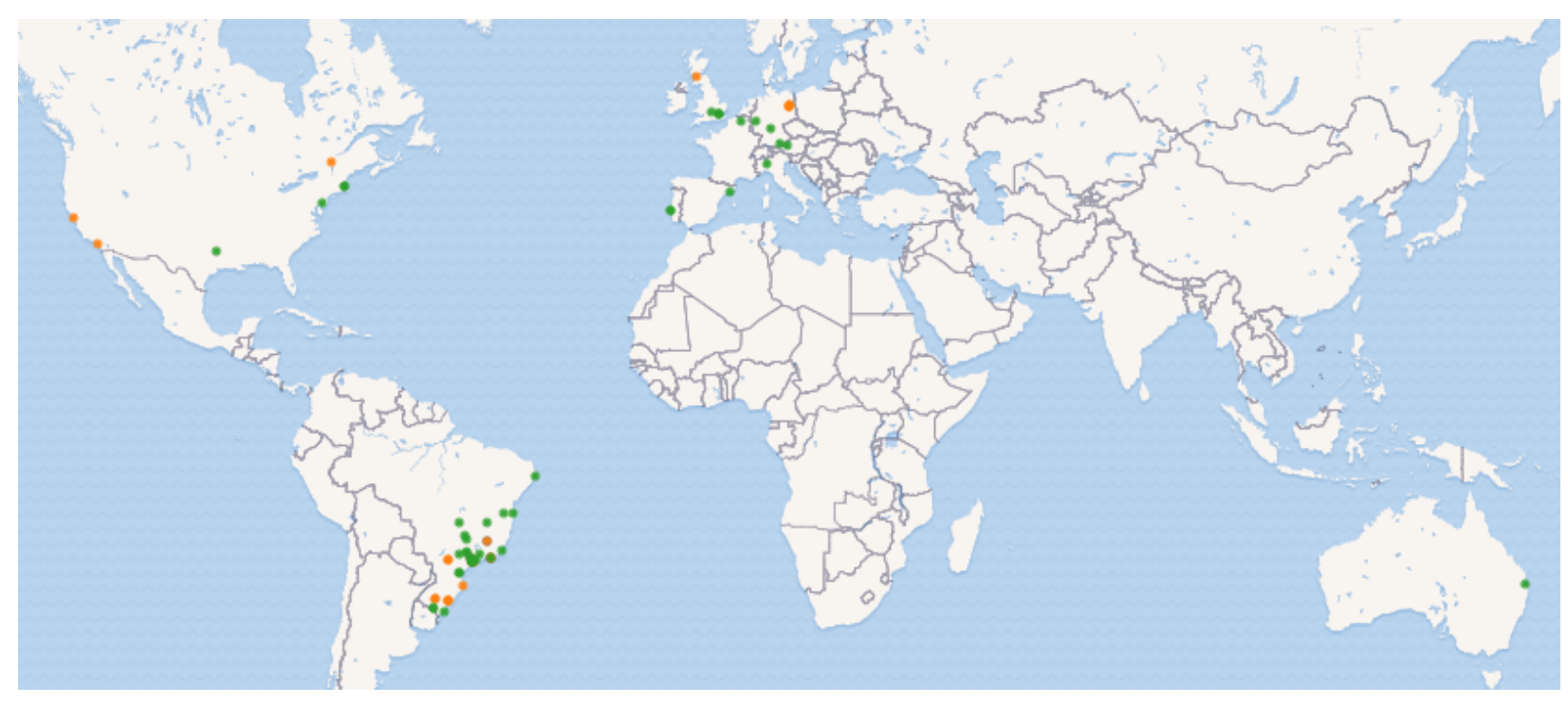

Figure 1: Map of affiliations of authors of papers that used RT-PCR. Coordinates and affiliation information were obtained automatically after open data from the Brazilian Reproducibility Initiative was merged to Wikidata. These are the only data available on Wikidata regarding the use of RT-PCR as of September 2021. An interactive version is available at https://w.wiki/45Ek. 
Information on authorship and affiliation was already on Wikidata: thus, all it took to build the map was to add the "missing" information to the database - i.e. the fact that those articles used RT-PCR.

In summary, thousands of systematic reviews are published each year [18], and a large part of the curation performed in the course of these reviews remains invisible, unusable, and unrecognized. Systematic reviews can be designed from the start to have their curated datasets in a format that makes them easy to find and use. We argue that this is an excellent opportunity for open science, where there is a lot to be gained from a little additional effort. If curated datasets are widely shared, distributed and combined, this could have a positive impact on the flow of scientific information in the life sciences.

\section{Acknowledgments}

TL is supported by grant \#2019/26284-1, São Paulo Research Foundation (FAPESP). OBA receives funding from FAPERJ (grant E-26/200.824/2021) and the Serrapilheira Institute. $\mathrm{KN}$ is supported by a scholarship from the Serrapilheira Institute.

\section{Author Contribution Statement}

TL, OBA and KN conceived the idea. TL and KN wrote the initial draft. TL, OBA and KN made critical revisions to the manuscript.

\section{References}

\section{The Brazilian Reproducibility Initiative}

Ana P. Wasilewska-Sampaio, Olavo Bohrer Amaral, Kleber Neves, Ana P. Wasilewska-Sampaio, Clarissa F. D. Carneiro, Olavo Bohrer Amaral, Clarissa F. D. Carneiro elife (2019-02-05)

https://www.wikidata.org/wiki/061799268

DOI: $\underline{10.7554 / e l i f e .41602}$

\section{PredictER}

Neal Haddaway, Martin Westgate https://estech.shinyapps.io/predicter/
3. Big data from small data: data-sharing in the "long tail" of neuroscience

Adam R. Ferguson, Jessica L. Nielson, Melissa H. Cragin, Anita Bandrowski, Maryann E. Martone

Nature Neuroscience (2014-11-01)

https://www.wikidata.org/wiki/024790499

DOI: $\underline{10.1038 / \mathrm{nn} .3838}$

4. When are researchers willing to share their data? Impacts of values and uncertainty on open data in academia

Stefan Stieglitz, Konstantin Wilms, Milad Mirbabaie, Lennart Hofeditz, Bela Brenger, Ania López, Stephanie Rehwald

PLOS ONE (2020-07-01)

https://www.wikidata.org/wiki/Q96836757

DOI: 10.1371/journal.pone.0234172

5. Google Dataset Search

https:// datasetsearch.research.google.com

6. Zenodo

https://zenodo.org/

7. figshare

https://figshare.com/

8. Dryad

https://datadryad.org/stash.

9. Identifiers for the 21st century: How to design, provision, and reuse persistent identifiers to maximize utility and impact of life science data

Julie A. McMurry, Nick Juty, Niklas Blomberg, Tony

Burdett, Tom Conlin, Nathalie Conte, Melanie Courtot, John Deck, Michel Dumontier, Donal K. Fellows, ... Helen Parkinson PLOS Biology (2017-06-29)

https://www.wikidata.org/wiki/033037209

DOI: $\underline{10.1371 / \text { journal.pbio.2001414 }}$

10. Wikidata

https://wikidata.org

11. Open by default: a proposed copyright license and waiver agreement for open access research and data in peer-reviewed journals

lain Hrynaszkiewicz, Matthew J Cockerill

BMC Research Notes (2012-09-07)

https://www.wikidata.org/wiki/Q21065691

DOI: $10.1186 / 1756-0500-5-494$ 


\section{Improving data access democratizes and diversifies} science

Abhishek Nagaraj, Esther Shears, Mathijs de Vaan

Proceedings of the National Academy of Sciences of the

United States of America (2020-09-08)

https://www.wikidata.org/wiki/Q99233710

DOI: $10.1073 /$ pnas.2001682117

\section{The citation advantage of linking publications to} research data

Giovanni Colavizza, Iain Hrynaszkiewicz, Isla Staden, Kirstie J. Whitaker, Barbara McGillivray

PLOS ONE (2020-04-22)

https://www.wikidata.org/wiki/Q93150448

DOI: 10.1371/journal.pone.0230416

14. Assessing scientists for hiring, promotion, and tenure.

David Moher, Florian Naudet, Ioana A. Cristea, Frank Miedema, John P. A. Ioannidis, Steven N. Goodman PLOS Biology (2018-03-29)

https://www.wikidata.org/wiki/052622119

DOI: 10.1371/journal.pbio.2004089

15. A data citation roadmap for scientific publishers Helena Cousijn, Amye Kenall, Emma Ganley, Melissa Harrison, David Kernohan, Thomas Lemberger, Fiona Murphy, Patrick Polischuk, Simone Taylor, Maryann E. Martone, Timothy W. Clark

Scientific Data (2018-11-20)

https://www.wikidata.org/wiki/059134700

DOI: $\underline{10.1038 / \text { sdata.2018.259 }}$

\section{Information about articles screened for the} Brazilian Reproducibility Initiative

Kleber Neves, Clarissa Carneiro, Ana Paula Wasilewska Sampaio, \& Pedro Batista Tan.

Zenodo (2021)

DOI: $\underline{10.5281 / \text { zenodo.4737506 }}$

\section{What is the most appropriate license for my data?}

Figshare

https://help.figshare.com/article/what-is-the-most-appro priate-licence-for-my-data
18. Wikidata as a knowledge graph for the life sciences Andra Waagmeester, Gregory Stupp, Sebastian Burgstaller-Muehlbacher, Benjamin M. Good, Malachi Griffith, Obi Griffith, Kristina Hanspers, Henning Hermjakob, Toby Hudson, Kevin Hybiske, ... Andrew I. Su eLife (2020-03-17)

https://www.wikidata.org/wiki/087830400

DOI: 10.7554/elife.52614

19. Are systematic reviews and meta-analyses still useful research? We are not sure.

Morten Hylander Møller, John P. A. Ioannidis, Michael Darmon

Intensive Care Medicine (2018-04-16)

https://www.wikidata.org/wiki/052584125

DOI: $10.1007 / \mathrm{s} 00134-017-5039-y$ 